\title{
El profesor como catalizador de energía emocional frente a la ambivalencia del nuevo entorno tecno-educativo'
}

Jesús Romero Moñivas"

\section{Resumen}

Este artículo pretende dos objetivos complementarios: por un lado, mostrar en el primer epígrafe las limitaciones constitutivas a los estudios teórico-empíricos que tratan de analizar los vínculos causales entre las tecnologías y la educación. Se intenta poner de manifiesto que tal empresa carece de un final feliz por sus propias limitaciones endógenas. Por otro lado, mostrar que, más allá de esos vínculos causales, hay una cuestión ineludible en el aspecto microeducativo: la co-presencia física de profesores y alumnos para generar rituales con alta energía emocional. Para ello, se utiliza el modelo de cadenas de rituales de interacción de Randall Collins (2004) y el modelo de autoeficacia docente de Pajares y Bandura. Se quiere poner de manifiesto que el papel del profesor universitario, además de la transmisión de información y contenidos (que exige un profesor con un papel activo en la investigación), es el de ser catalizador y generador de energía emocional en el aula, que requiere la copresencia física. Se concluye que dependiendo de los objetivos de los estudiantes a la hora de elegir sus estudios en cuestión, estos podrían virtualizarse completamente cuando sólo se busca la adquisición de contenidos, o deberán mantener la co-presencia física cuando los objetivos del estudiante rebasen lo puramente informativo, y busquen también la entrada en rituales de interacción en los que se transmitan emociones y energías que coadyuvan a la importancia de los contenidos intelectuales.

\section{Palabras clave}

Autoeficacia docente - Energía emocional - Tecnología y educación Micro-rituales educativos - Enseñanza universitaria.
I- Este trabajo se encuadra dentro del Proyecto La institución escolar en la era de la información: entre el aula sin muros y la educación sin escuelas (CSO201238678), subvencionado por el Ministerio de Economía y Competitividad (3 años). II- Universidad Complutense de Madrid, Madrid, España.

Contacto: jesus.romero@edu.ucm.es 


\title{
Professors as emotional energy catalysts in the face of the ambivalence of the new techno-educational context'
}

Jesús Romero Moñivas"

\begin{abstract}
In this paper, I aim to achieve two complementary objectives. Firstly, to show the constitutive constraints to the theoretical and empirical studies which analyze the causal links between technology and education. I seek to evidence that such endeavor lacks a happy ending due to its own endogenous limitations. Secondly, I aim to evidence that, beyond these causal links, there is an inevitable issue in the micro-educational dimension: the physical co-presence of professors and students to generate successful rituals with high emotional energy. To this end, I use the interaction ritual chains by Randall Collins and the model of teacher self-efficacy by Pajares and Bandura. Thus, I stress that, besides transmitting information and content (which requires professors with active roles in research), the role of university professors is to be catalysts and generators of emotional energy in the classroom, which also requires their physical co-presence. I conclude that, depending on the objectives of the students when choosing their studies, these could be fully virtualized when students seek only content acquisition, or professors' physical co-presence should be maintained when students' goals go beyond purely informational acquisition and seek interactional rituals which transmit emotions and energy that contribute to the importance of intellectual content.
\end{abstract}

\section{Keywords}

Professor's self-efficacy - Emotional energy - Technology and education - Education micro-rituals - University education.

\footnotetext{
I- This study is part of the project La institución escolar en la era de la información: entre el aula sin muros y la educación sin escuelas (CSO201238678), funded by Ministerio de Economía y Competitividad (three years). II- Universidad Complutense de Madrid, Madrid, España.

Contact: jesus.romero@edu.ucm.es
} 
Tecnología y educación: teorías claras y distintas para una relación oscura y confusa

Hace ya unas décadas el discurso sobre la educación es dominado por un conjunto de problemas que suele encuadrarse bajo el genérico paraguas de tecnología y educación. Pueden distinguirse dos clásicos bandos respecto a cualquier sistema socio-técnico: los optimistas y con tendencia a ver lo bueno (integrados o tecnófilos), y los más pesimistas $\mathrm{y}$ que manifiestan una inclinación a resaltar los aspectos más oscuros de lo tecnológico (apocalípticos o tecnófobos). Estos dos bandos están constituidos por representantes de todas las ciencias sociales, desde las más directamente vinculadas con la educación o la pedagogía, a disciplinas más alejadas como la filosofía. Entre medias, sociólogos, psicólogos, antropólogos, teóricos de la comunicación, economistas, etc. En general, la literatura teórica y empírica se ajusta a esa dicotomía, aunque somos cada vez más los que pensamos que la realidad se encuentra en un punto medio, caracterizado por la ambigüedad, la oscuridad y la ambivalencia. Las conclusiones tecnófilas o tecnófobas son la mayor parte de las veces demasiado aventuradas y muy sesgadas por los puntos de partida ideológicos de los investigadores: si uno quiere encontrar perniciosa la tecnología para la educación, encontrará los datos que lo confirmen; si se quiere defender la utilidad de la tecnología, también habrá datos disponibles ${ }^{1}$.

Parece que lo más adecuado es un punto medio, pero no deliberadamente neutral con la intención de acomodarse en la ambigüedad y eludir los fuegos cruzados del debate. Una zona media es el mejor punto de partida de una ciencia rigurosa y serena, alejada de los aspavientos y de los compromisos a priori. No sólo porque es una ley de las ciencias sociales

1- Aunque he utilizado deliberadamente la dicotomía entre tecnófobos y tecnófilos, en realidad he mostrado en otro lugar (ROMERO MOÑIVAS, 2015) que esas etiquetas son abstracciones infundadas, y que lo que sucede en realidad es que las personas mostramos ambivalencias situacionales respecto a la tecnología. el hecho de que se necesita perspectiva histórica para poder valorar los fenómenos humanos, sino porque me parece que respecto a este confuso binomio de tecnología (¿qué es la tecnología?) y educación (¿qué es la educación?) encontramos una serie de limitaciones demasiado serias para que realmente pueda dirimirse el conflicto y pueda demostrarse que real y efectivamente la tecnología mejora o empeora la educación. Por ello, a menudo se hace ciencia dogmática de un objeto de estudio que es demasiado ambivalente, ambiguo y con un contorno poco claro. No hay cartesianismo posible para esta cuestión; no hay ideas claras y distintas que expliquen lo que en realidad es una relación oscura y confusa.

Como un intento de reflexión sobre este oscuro binomio educación-tecnología, quisiéramos tratar dos temas relacionados. En la primera parte, se exponen las razones que limitan los estudios actuales de las relaciones entre la tecnología y la educación. En la segunda parte, tratamos uno de los problemas esenciales de la educación frente a la ambivalencia de los nuevos entornos tecnoeducativos: el papel fundamental del profesor en la transmisión de Energía Emocional. Aunque son dos grandes partes argumentativamente independientes entre sí, ambas están esencialmente conectadas por una razón de fondo: la primera parte muestra lo problemático que es estudiar las relaciones ambivalentes entre educación y tecnología. En la segunda, recogiendo esa enseñanza teórica, rescatamos la idea de la necesidad de la emergencia de rituales exitosos dentro del aula, para lo que el profesor debería de ser un catalizador de Energía Emocional, independientemente de los juicios sobre el ambivalente entorno tecno-educativo.

\section{Limitaciones de los estudios sobre las relaciones tecnología y educación}

\section{Limitaciones respecto a la conceptualización de la tecnología}

En este aspecto, el siglo de investigación teórico-empírica de los historiadores, 
sociólogos y filósofos de la tecnología nos da algunas claves:

(a) La tecnología (no) es exclusiva de las sociedades modernas. Consciente o inconscientemente, en la mayor parte de la literatura sobre tecnología y educación se comete el error de dar por hecho que la tecnología es exclusiva de las sociedades actuales. Evidentemente, la tecnología digital e informática lo es, pero con ello se pasa por alto que siempre ha existido una tecnología relacionada con un modo de entender la educación. Por tanto, no es una novedad histórica el binomio entre artefactos y educación, al contrario: la historia muestra que constitutivamente ambas siempre han ido juntas, con sus luces y sus sombras.

(b) Asimetría entre lo social y lo tecnológico. Otro error es contraponer tecnología y sociedad (o educación) como dos ámbitos ontológicos diferentes. Sin embargo, la tecnología no nace, se desarrolla y difunde en un vacío social, sino que como han puesto de manifiesto la perspectiva contextualista (STAUDENMAIER, 1985) en historia, y la constructivista (PINCH; HUGHES; BIJKER, 1997) y la teoría del actor-red (LATOUR, 1992; LAW, 1992) en sociología, los procesos de innovación y desarrollo tecnológicos son procesos esencialmente sociales. Que el funcionamiento y construcción de artefactos sean asuntos técnicos, no significa que sean sociológicamente neutrales. Las luchas de poder entre grupos con proyectos de diseño de la misma o parecida tecnología, pone de manifiesto que ningún artefacto es neutral sino que lleva inscrita una visión del mundo más o menos conscientemente buscada, y por ello la construcción de los sistemas sociotécnicos pertenece a las propias entrañas de la construcción social de la realidad (RAMMERT, 2002; DEGELE, 2002), porque la tecnología no es lo otro distinto de la sociedad (JOERGES, 2001). Esto implica que mucho antes de analizar qué posibles influencias tiene la tecnología sobre el ámbito educativo o de qué modo las personas asumen esas tecnologías, es necesario preguntarse qué concepción del mundo y qué pedagogía se encuentra cristalizada en el propio diseño de esas tecnologías educativas.

(c) Las tecnologías (no) son esencialmente artefactos técnicos. Otro de los errores es considerar que la tecnología es el artefacto discreto y concreto, como una pizarra digital o un ordenador. Las tecnologías nunca son objetos concretos aislados, sino que vienen entretejidos en sistemas socio-técnicos (HUGHES, 1997) o redes con otros artefactos, con seres humanos, con animales, con leyes, con patrones culturales, incluso con objetos inanimados como electrones u otro tipo de partículas (LATOUR, 1997). Piénsese en la electricidad o en un aeropuerto, pero también en lo que suponen las Tecnologías de la Información y la Comunicación (TIC) y el nuevo mundo digital. Es precisamente por este entretejimiento sistémico el motivo por el que se puede hablar de cierto determinismo tecnológico o, como lo llama Hughes (1996), momentum o inercia de los sistemas socio-tecnológicos cuando adquieren un gran tamaño y mayores vínculos con otros elementos del sistema. Es evidente que un ordenador por sí sólo no determina la sociedad ni la vida de un individuo, ni tampoco al sistema educativo. Pero si a ese ordenador le sumamos la nueva cultura digital, las normativas legales sobre gestiones a través de ordenadores y toda la miríada de pequeños artefactos que van asociados al sistema informático, ya no estamos hablando de un objeto concreto, sino de todo un entramado socio-técnico que se ha solidificado en el conjunto de la sociedad. La tecnología en abstracto no existe, igual que no existe la educación en abstracto, sino sólo los entramados socio-técnicos. Lo que se contraponen no son nuevos objetos concretos a otros viejos objetos concretos dentro del sistema educativo, sino sistemas socio-técnicos con unos objetos, unos determinados conocimientos, unos valores, unas culturas, unos objetivos, unas visiones del mundo, etc. a otros sistemas socio-técnicos alternativos, incluso aunque a veces compartan algunos de los mismos artefactos tecnológicos. 
(d) La influencia de la tecnología sobre el ámbito no técnico (no) es directa $y$ uni-direccional. Finalmente, tanto los defensores como los críticos de las TIC en el ámbito educativo comparten en su mayoría la presuposición determinista de que la tecnología influye directamente en la educación, olvidando no sólo la realidad de los sistemas socio-técnicos, sino los procesos de apropiación social de la tecnología, que tiene ritmos propios y a menudo autónomos de los cambios tecnológicos.

\section{Limitaciones respecto al vínculo experimental tecnología-educación}

Hay un segundo conjunto de limitaciones que afecta radicalmente al vínculo experimental de los estudios, tanto optimistas como apocalípticos, que buscan los datos que confirmen sus posturas. Las limitaciones de tipo experimental tampoco son fácilmente eliminables con la mejora de los diseños técnicos, puesto que el problema reside en la ontología de fondo que subyace a esos diseños:

(a) Desde un punto de vista metodológico no es fácil discriminar la variable tecnología de otro tipo de variables que entran en juego dentro del éxito o del fracaso escolar, y más problemático aún es afirmar que existe causalidad y no meras correlaciones, incluso espurias. Los estudios empírico-cuantitativos que tratan de buscar correlaciones entre variables no dejan de caer en el peligro tantas veces advertido, de la artificial parcelación de la realidad en variables dependientes e independientes, abstracción hecha de la complejidad intrínseca de la ontología social. Ciertamente, el desarrollo de los métodos estadísticos hace posible tales discriminaciones de variables discretas, pero como gran parte de la sociología cualitativa y crítica ha puesto de manifiesto, el problema fundamental es que aunque metodológicamente es posible tal separación puede, aun así, suponer una traición ontológica y epistemológica a la complejidad de la realidad social. (b) Desde un punto de vista epistemológico es un absurdo gnoseológico evaluar en abstracto el éxito o el fracaso escolar, según un tipo u otro de tecnología. Este concepto ha cambiado a lo largo de la historia y además es multidimensional. En los estudios históricolongitudinales, no tiene sentido afırmar que la existencia de un ordenador por cada niño en un aula les hace tener más éxito que a los niños que vivían a mediados de los años cincuenta del siglo XX, en el siglo XIX o en la antigua Grecia, por ejemplo; y en los estudios sincrónicos la argumentación se puede convertir en una falacia ideológica si no se explica qué criterio se está utilizando para medir el éxito o el fracaso: la memoria, la capacidad creativa, la resolución de problemas, la tendencia crítica, etc. Es dudoso que una tecnología, sea cual fuere, pueda correlacionar negativa o positivamente con todos y cada uno de esos criterios. Ciertamente, las conclusiones de los estudios más actuales tienen cuidado en afirmar que sus correlaciones se refieren a competencias clásicas o digitales, etc. Pero precisamente esto nos lleva a la duda de si acaso tiene algún valor ese tipo de conclusiones, más allá de que evidentemente el lápiz no es un buen artefacto para adiestrar en la escritura digital, y que el uso masivo del almacenamiento en un ordenador o el acceso a la información en la red con un sólo click quizá sea una mala tecnología para educar la capacidad de la memoria.

(c) Finalmente, desde un punto de vista ontológico permanece el problema general de qué entendemos por tecnología, puesto que el ser humano desde sus orígenes es esencialmente un ser entre objetos, y cada objeto o red de objetos ha exigido -desde las hordas primitivas- un conocimiento y una adaptación cambiante del aprendizaje. Por ello, si lo que se quiere afirmar es que en un mundo donde domina el ordenador tendrá más éxito quien sabe manejarlo mejor, no son necesarios estudios empíricos, puesto que lo mismo se podría decir de las sociedades en las que predominaba la lanza, la azada, el estribo, el cañón, el libro o la bicicleta. Por el contrario, 
si lo que pretende afirmarse es que los niños que manejan tabletas digitales ya no querrán leer en libros de papel, o que el acceso a Internet puede distraer su atención de lo realmente importante, habrá que preguntar qué es lo importante y si en el mundo futuro eso seguirá siendo tan importante o pasará a tener la relevancia que, actualmente, tiene el conocimiento de la técnica de ordeño de vacas para un ciudadano de las megaurbes. No es fácil ironía, es más bien un problema de calado teórico. Esta es ciertamente la limitación más importante de las tres, puesto que es de donde brotan las diversas posturas en relación con todo el entramado socio-técnicoeducativo. Si no se explicita de forma clara qué tipo de conocimiento, qué modo de entender la educación y qué manera de aprehender la realidad se tiene, difícilmente puede hacerse ciencia rigurosa del conjunto del ecosistema tecno-educativo. Pero seguirán compitiendo paradigmas socio-técnico-educativos diferentes sin poder dirimirse empíricamente cual es superior a los demás.

\section{Limitaciones respecto a la multi-dimensionalidad del objeto de estudio}

Hay otras limitaciones que se refieren a la confusión de dimensiones. La sociología tiene necesariamente una epistemología situacional. En el ámbito de lo histórico y lo social la comprensión de un fenómeno debe situarse siempre en referencia a un contexto o a una situación. Esto supone rebajar el tono abstracto y universalizante de nuestras afirmaciones, relativizándolas a los contextos y situaciones donde adquieren sentido. Es innegable que la mayor parte de los estudios empíricos han ido refinando este aspecto tratando de discernir esos diversos niveles. Ahora bien, desde un punto de vista teórico es muy frecuente pasar de determinados estudios empíricos situacionales a generalizaciones más apresuradas.

Todo fenómeno social puede analizarse desde una dimensión micro, meso o macro. Obviamente, en el caso concreto que nos ocupa, es evidente que no es lo mismo la dimensión micro, por ejemplo, de los micro-rituales de los docentes, los discentes o la relación mutua entre ambos. Puede suceder que algunos autores atribuyan bondades a las tecnologías cuando se trata de enseñar (pizarras digitales), pero que consideren que otras tecnologías pueden distraer la atención de los estudiantes en sus casas (redes sociales), y otras que son ambiguas cuando vehiculan la relación profesor-alumno en un aula (correo electrónico, etc.). En la dimensión meso sucede lo mismo: los centros deben estar equipados con unas tecnologías, pero no con otras; o subgrupos de profesores, de padres y de alumnos, insisten en que sus centros deben estar bien surtidos tecnológicamente, pero no se ponen de acuerdo sobre cuales son los utensilios, cómo deben utilizarse y qué franja temporal de uso debe exigirse, así como qué tipo de contenidos y metodologías deben privilegiarse. Finalmente, hay otros factores de dimensión macro en los que suele coincidirse de manera más unánime: tanto tecnófilos como tecnófobos suelen estar de acuerdo -quizá por la propia evidencia histórica al respecto (TASSEY, 2007)- en que el país debe invertir en investigación y desarrollo, y que sólo aquellos países con competitividad tecnológica pueden tener crecimiento económico y generar empleo; de ahí que inversión en educación e inversión en tecnología parecen estar en armonía en la dimensión macro, mientras no se descienda a lo concreto. En todo caso, la dimensionalidad y contextualidad del fenómeno tecnológico refuerza las limitaciones constitutivas de los estudios empíricos basados en correlaciones entre artefactos técnicos y educación.

\section{Limitaciones respecto a la claridad y distinción del objeto de estudio}

Lo que hemos expuesto hasta aquí supone que difícilmente puede dirimirse el conflicto entre los diversos bandos y posturas respecto a las relaciones entre la tecnología y la educación, debido a limitaciones que son 
constitutivas al objeto de estudio. Hay una suerte de incompletitud que impide formular teorías claras y distintas con carácter apodíctico. La ambivalencia es más cercana a la realidad (FERNÁNDEZ ENGUITA, 2013). Hemos insistido recientemente (ROMERO MOÑIVAS, 2013b) que el debate en torno a tecnófilos y tecnófobos respecto a la educación es una simplificación grosera de un lucha más compleja, con diversos bandos, con promiscuidades ambivalentes entre ellos y que obedece más bien a concepciones de fondo alternativas, irreconciliables y, por lo mismo, inconmensurables. No habrá datos científicos que puedan cortar el nudo gordiano de la lucha, de ahí que no puedan sacarse conclusiones propositivas y normativas de estudios puramente descriptivos en los que, además, se incurre en todas las limitaciones anteriores.

No hay dos bandos enfrentados, sino que hay un espectro mucho mayor que supone que cada grupo en conflicto tiene tres frentes de ataque/defensa en torno a los cuales tiene que hacer elecciones: el tipo de conocimiento, el tipo de redes tecnológicas y el tipo de sistema de organización-metodología de la educación. Por ello, habrá tantas posibilidades como combinaciones diferentes entre esas distintas variables. No hay una sola dirección, sino una rejilla de posibles alternativas. No es obligatorio aceptar el paquete completo: uno puede querer que su conocimiento sea de un tipo o de otro, sin que eso suponga aceptar que tengan que cambiar las tecnologías o las organizaciones de la educación a través de las cuales aprender ese conocimiento. Por ello, decir que ciertas tecnologías fomentan o inhiben determinadas competencias (sean clásicas o digitales), no puede hacerse en un vacío abstracto, en una supuesta neutralidad objetivo-científica. No hay un enfoque funcional o neutral que nos diga cual será el mejor arreglo socio-técnico-educativo del futuro, sino que hay un conflicto relativista en el que los intereses enfrentados construyen sus realidades legitimándolas con datos científicos. La historicidad y situacionalidad humana es, en este punto, inquebrantable e insuperable epistemológicamente.

\section{Energía emocional en medio de los micro-rituales en el aula}

Con esa conclusión de fondo, en esta segunda parte nos centraremos en exponer lo que consideramos (por tanto, es nuestra elección dentro de este conflicto relativista) que es un rasgo importante de la educación: la creación de rituales educativos exitosos con altos niveles de energía emocional. En la primera parte ha quedado claro que en cierto modo es estéril entrar en luchas sobre si los nuevos entornos sociotécnicos son educativamente mejores o peores. A pesar de los esfuerzos empíricos no es posible una respuesta que no sea estrictamente situacional y cargada de interpretaciones a priori. Sin embargo, creemos que hay un rasgo importante de los nuevos entornos tecno-educativos que nos gustaría tener en cuenta aquí: la posibilidad de una virtualización completa de la enseñanza a través de plataformas digitales totalmente online (que transforma tecnológicamente lo que a mediados del siglo XX era simplemente educación a distancia) y sus consecuencias. La enseñanza clásica a distancia y la moderna online tienen un papel fundamental en la expansión de la movilidad psíquica moderna gracias al mayor acceso de más capas de población a la información y el conocimiento. Por ello es miopía histórica rechazar las mejoras que la enseñanza a distancia y virtual han supuesto en el último siglo. Como bien advirtió ya hace cuatro décadas Peters (1998, 2004), el nacimiento de la educación a distancia toma como modelo los procesos industriales, y sólo una sociedad industrial pudo generar la educación a distancia, de la misma forma que sólo una sociedad postindustrial ha podido forjar una educación online virtualizada. El gran acierto de la teoría de Peters es que reconoce las peculiaridades propias de la educación a distancia y/o online, su carácter sui generis, en el que muchos de los métodos tradicionales de interacción cara a cara quedan 
obsoletos y sin sentido. En realidad, ese tipo de enseñanza no es simplemente otro modo de enseñar, sino que a él subyace otro modo de comprender el conocimiento, es otro mundo pedagógico diferente. Por este motivo, nuestro interés no es estudiar la enseñanza virtual o a distancia per se, sino reflexionar sobre un hecho aparentemente evidente, pero a menudo no explicitado: es más difícil que a través de la virtualización (o la clásica educación a distancia) se puedan generar rituales emocionales entre profesores y estudiantes. Por ello, habrá que situar en su contexto, qué se busca en concreto con cada tipo de educación: presencial, semipresencial, a distancia y virtual. No obstante, aquí sólo se quiere defender la ineludible importancia de la co-presencia física en una educación universitaria que no quiera ser simplemente una transmisora de información. Para ello recurriremos a la teoría ritual de Randall Collins (2004).

\section{El concepto de ritual de interacción y de Energía Emocional (EE)}

En sociología se distingue entre interacción e interdependencia. La primera hace referencia a las interacciones micro-situacionales en las que la co-presencia física y la corporalidad son elementos necesarios y constituyentes, en tanto que en la interdependencia (ELIAS, 1970; ROMERO MOÑIVAS, 2013a) las relaciones sociales se amplían para incluir aquellas cadenas de relaciones macro que vinculan a las personas más allá de una relación estrictamente física. Pero ¿qué importancia tienen los micro-rituales físicos para la educación? Para responder expondremos, en primer lugar, el concepto de ritual de interacción; en segundo lugar, explicaremos el constructo teórico de la autoeficacia docente, con el objetivo de relacionar ambas cosas.

El punto de partida es la constatación de que las personas tienen multitud de interacciones cara a cara a lo largo de su día y de su vida, unas influyendo en otras, en una cadena continua que se retroalimenta. Collins ha elaborado una teoría de las cadenas de rituales de interacción (RI), que en su forma más acabada puede resumirse en cuatro ingredientes o condiciones iniciales (1-4), y cuatro resultados (4-8) (COLLINS, 2004). Los ingredientes: (1) Reunión física de participantes, (2) barreras que excluyen a los outsiders, (3) foco mutuo de atención y (4) un tomo emocional compartido. Los resultados: (5) sentimientos de afiliación al grupo, (6) objetos sagrados o emblemas simbólicos, (7) Energía emocional y (8) sentimientos morales de lo correcto e incorrecto (ROMERO MOÑIVAS, 2013c).

Por lo tanto, un ritual de interacción es una reunión física de personas que desarrollan un foco de atención y que entran en un ajuste mutuo de micro-ritmos corporales y emociones. Existen dos grandes tipos de rituales: por un lado, los rituales naturales en los que hay una carga menor de procedimientos estereotipados, y por otro, los rituales formales, que se inician con un complejo de procedimientos ceremoniales y que se basan en una codificación más estricta de las diferentes actuaciones de las personas implicadas en ellos. No es lo mismo un ritual de dos amigos que se encuentran en la calle o un ritual de defensa de tesis doctoral. Ambos pueden ser rituales exitosos o fracasados, es decir, pueden conseguir o no generar las emociones unificadas que pretendían (COLLINS, 2004). En el caso del ritual que se desarrolla en un aula entre el profesor y sus estudiantes, es una mezcla de elementos protocolarios y naturales; no obstante, una tesis básica de este artículo es que el profesor tendrá más éxito en la generación de un ritual exitoso con los estudiantes cuanto más natural y menos formal sea (o parezca) su actuación en el aula, dando por hecho que desde un punto de vista sociológico no hay nunca un ritual absolutamente espontáneo y natural sin cierta carga de construcción y actuación (GOFFMAN, 1994); mientras que la enseñanza virtualizada tenderá a ser más protocolarizada, $\mathrm{y}$ por lo mismo más formal. De hecho, el concepto de distancia transaccional de Moore (2005, 
2007) implica que la brecha de comunicación e incomprensión que se genera por la separación física de la educación a distancia, sólo puede ser minimizada aumentando las variables diálogo y estructura: es decir, la relación profesor-alumnos y la organización formalizada del curso.

Independientemente de ello, hay dos elementos básicos para comprender la aplicación sociológica de los rituales de interacción: (1) Para Collins (2004) la corporalidad y el foco mutuo de atención en la sintonización (entrainment) es un requisito indispensable para un ritual, puesto que aumenta la intensidad de las emociones compartidas y el ajuste de los micro-ritmos de la interacción, y señala y confirma el foco de atención. Por ello, los diversos encuentros físicos tanto en rituales de diversión como de condolencia, así como las celebraciones deportivas e, incluso, la transmisión de la cultura o la enseñanza en la formación oficial, son esencialmente rituales que requieren co-presencia física, aunque los nuevos medios electrónicos hagan posible la ritualización a distancia, pero con resultados de menor nivel de intensidad, solidaridad y sacralidad de los símbolos. La corporalidad, en definitiva, facilita la hiper-sintonización de los ritmos corporales y emocionales entre las personas y su atención en un foco compartido. $+$

\section{La autoeficacia del profesor y su Energía Emocional}

Nuestra visión del profesor como catalizador de Energía Emocional encuentra otro asidero teórico-empírico en los estudios de autoeficacia (self-efficacy) docente de autores como Frank Pajares (1996), Albert Bandura (1977) y, en España, por ejemplo Leonor Prieto Navarro (2007). La autoeficacia docente -un constructo teórico que, siguiendo a Bandura, se define como "los juicios de cada individuo [profesor] sobre su capacidad, en base a los cuales organizará y ejecutará sus actos de modo que le permitan alcanzar el rendimiento deseado" (1977, p. 193) - tiene repercusiones sobre el modo en que el profesor enseñará y, por lo tanto, influirá en el aprendizaje del alumno. Por este motivo, conocer el modo en que los profesores perciben su capacidad de enseñar, de influir en los alumnos, sus creencias pedagógicas, etc., es necesario para mejorar la docencia y el aprendizaje.

En este sentido, es habitual entre los teóricos de la educación considerar un conjunto básico de rasgos que tanto alumnos como profesores consideran como características necesarias para la alta calidad de un profesor universitario: 1) Conocimiento de la materia; 2) Habilidades o destrezas pedagógicas; 3) Relaciones interpersonales; 4) Relación docencia-investigación; 5) Características de personalidad. La investigación y el dominio de la materia están más relacionados con la cuestión de la transmisión de contenidos. Es común que incluso un alumno de primer curso de carrera sepa distinguir un profesor actualizado, informado, que investiga, que publica libros y que, por lo mismo, es capaz de transmitir un contenido de mayor calidad. Ahora bien, esta pura transmisión de información o contenido es sólo una de las dimensiones de lo que se considera ser buen profesor. La otra dimensión tiene que ver con lo que hemos llamado ser un catalizador de energía emocional y que incluiría las habilidades pedagógicas, las relaciones interpersonales y los rasgos de personalidad (aunque estos últimos podrían quedar fuera del modelo). Este segundo conjunto de habilidades no tiene que ver tanto con el contenido que se transmite, sino con la capacidad emocional de transmitir no sólo esos contenidos, sino cosas más intangibles como la motivación, la pasión de lo que se dice, la confianza en los alumnos y cierto liderazgo interpersonal. Lo emocional y pasional es también un rasgo esencial de toda excelencia docente $y$, por ello, ambas dimensiones son igualmente necesarias: difícilmente se podrá ser buen profesor universitario si se conocen muy bien los contenidos y se investiga mucho, pero falta la comunicación físico-emocional de los aspectos interpersonales. Pero tampoco lo será aquel que con destrezas comunicativas y 
emocionales excelentes, carece, sin embargo, de un equilibrio entre docencia-investigación así como el dominio de los contenidos. De hecho, los estudios neurológicos han venido insistiendo en este contexto emocional de toda racionalidad humana sana y normal desde el clásico estudio en los años noventa de Antonio Damasio (2006). En el ámbito hispanohablante el neurólogo Francisco Mora ha insistido repetidamente que los hechos son ciegos para el cerebro si no pasan por la interpretación emocional del sistema límbico (MORA, 2014). De ahí que afirme repetidamente en los medios de comunicación sólo puede aprenderse aquello que se ama.

Es instructivo a este respecto que el propio Randall Collins (1998) en su teoría sobre las dinámicas de las redes intelectuales, constate que las grandes figuras intelectuales siempre proceden del discipulado de otras grandes figuras. Estas redes verticales tienen un sentido muy claro: a través de ellas, de los maestros a los discípulos, circulan tres mercancías: energía emocional, capital cultural y conocimiento de la estructura de oportunidad del campo intelectual. Si desechamos la última por ser más propia de las relaciones que tienen que ver con lo académico en su dimensión de carrera docente e investigadora, es significativo que las otras dos grandes mercancías sean, precisamente, las dos dimensiones a las que se hacía referencia más arriba. Los grandes profesores no sólo transmiten capital cultural o energía emocional apasionada, sino ambas a un tiempo.

Se reproduce la clásica dicotomía entre el mero profesor y el maestro, entre el decir y el mostrar. Wittgenstein en su Tractatus precisamente juega con esta doble dimensión entre lo que puede ser dicho (sagen) y lo que puede ser mostrado (zeigen), lo que pertenece al mundo lógico-argumentativo y lo que pertenece al mundo del sentido (Mèlich, 2010). Lo que puede ser dicho siempre está vinculado a lo que tiene significado, es decir, lo que puede ser dicho pertenece a la esfera de lo racional e informativo. En tanto que aquello que puede ser mostrado pertenece al ámbito del sentido, de lo que sólo puede aprenderse a través de la convivencia entre personas, en compañía corporal, a través del ejemplo humano. Por ello, la dicotomía entre decir y mostrar no tiene nada que ver entre lo que puede ser hablado o visto. De hecho, un experimento químico es algo que se ve, pero pertenece por su cualidad informativo-objetiva al ámbito de lo dicho; se puede aprender un experimento en un video de Youtube sin necesidad de convivir con el químico. En realidad, los contenidos pueden ser transmitidos, pero la Energía Emocional sólo puede ser mostrada y transmitida en medio de la co-presencia física de los rituales de interacción entre profesor-alumno. Un mero transmisor de información es un profesor erudito, un mero transmisor de energía emocional es un profesor de buen rollo, un transmisor de ambos es un maestro. En realidad, para que exista un ritual de interacción exitoso dentro de un aula, el profesor debería de manifestar esa doble cualidad.

La Energía Emocional del profesor no es un mero poseer para sí mismo, sino que es una de las mercancías que se transmite junto al capital cultural. A menudo el fracaso del ritual educativo (que puede traducirse en suspenso, en absentismo en el aula, en distracción, en falta de motivación, etc.) tiene que ver precisamente con que el alumno no recibe la suficiente EE del profesor, bien porque este no la posee él mismo o porque no es capaz de generarla colectivamente. Por este motivo, la virtualización completa (no hablamos de simple mediación técnica) de la enseñanza no es útil cuando lo que se pretende es generar rituales de interacción exitosos. Las TIC son un instrumento muy adecuado para la transmisión a distancia de lo que puede ser dicho, pero no de lo que puede ser mostrado, de ahí su ambivalencia constitutiva. De hecho, el malestar clásico de Holmberg (2003), desde los años 60, hacia la educación a distancia y la actual online reside precisamente en su percepción de que a menudo este tipo de enseñanza sólo se centra en la adquisición de información de 
manera solitaria. No es casual que él mismo considere que es necesario introducir elementos como la empatía, la conversación amistosa y la interacción continua entre el profesor y sus estudiantes. En realidad Holmberg detecta que la co-presencia física transmite algo que no es informativo, sino emocional-personal. Ahora bien, el problema es que ciertamente en la educación online y a distancia se puede disminuir la deficiencia emocional con esas estrategias comunicativas, pero nuestro argumento es que no pueden hacerlo con la misma fuerza e intensidad que en la enseñanza co-presente.

Por supuesto, no todas las relaciones sociales exigen co-presencia física. De la misma forma, no todos los objetivos educativos exigen rituales de co-presencia física: si el objetivo educativo de una persona es la adquisición de información, de lo que puede ser dicho, entonces la virtualización digital es un modo excelente, rápido, descentralizado y ubicuo para adquirirlo. Leer libros o navegar por la red es quizá la mejor forma de acumular información y conocimiento, e incluso destrezas técnicas (abundan los cursos virtuales de mecanografía e idiomas). Ahora bien, es significativo a este respecto, que los propios intelectuales, a pesar de lo fácil que sería mantener redes académicas de transmisión de información a través de Internet, el intercambio de libros, artículos, etc., no han dejado de mantener rituales de co-presencia desde sus mismos orígenes: sigue habiendo cientos de congresos cada año, seminarios, reuniones de grupos de trabajo, jornadas, etc. La explicación es suficientemente intuitiva, y nos la da el propio Collins (2004): en esos encuentros físicos y corporales se transmite algo más que contenido intelectual e informativo; se transmite EE para seguir en la lucha académica, se transmite pasión por el campo de estudio, y con ello se generan rituales de interacción que pueden elevar la EE tanto de los novatos académicos como de los gurús; esa recarga de EE permite a los intelectuales que después, durante la mayor parte del año, pasen largas horas solos, sentados en sus ordenadores y rodeados de libros.

Así, pues, tener alta autoeficacia -según Pajares (1996) y Bandura (1977)- es muy semejante a tener alta Energía Emocional, según el modelo de Collins (2004). La diferencia entre los profesores con alta autoeficacia y alta EE radica, precisamente, en que sienten que son capaces de transmitir algo más que pura información ${ }^{2}$. Los estudiantes perciben y reciben esto, produciéndose un contagio emocional entre ellos. Ciertamente, la EE puede ser almacenada en símbolos que periódicamente alimenten de nuevo a los sujetos. Pero ese almacenamiento es subsiguiente al ritual generador. Así, un aula se puede convertir en un ritual exitoso, cuando profesor y estudiantes salen de él con elevados niveles de EE, motivado uno respecto a su trabajo y motivados los otros respecto a sus estudios. La fuerza del ritual deriva en gran parte de ese carácter corporal y físico del encuentro. La carga emocional es más difícilmente alimentada de forma vicaria a través de medios tecnológicos a distancia, aunque técnicamente se haya podido redefinir la espacialidad clásica.

Esta concepción de la enseñanza en co-presencia no es puro romanticismo trasnochado. Al contrario, la fundamentación neurofilogenética es importante a este respecto. Los seres humanos somos más semejantes a los solitarios simios (ape) que a los gregarios monos (monkey), debido a que carecemos de bioprogramadores que naturalmente nos faciliten la construcción de vínculos sociales. Estos vínculos sociales que son, sin embargo, esenciales para la vida humana se producen trabajosamente,

2- También es cierto que en muchas ocasiones un profesor puede sentir baja autoeficacia o baja EE precisamente porque no domina los contenidos teóricos de la asignatura y se siente vulnerable. No obstante, este rasgo suele ser pasajero: bien por la juventud del docente, bien porque circunstancialmente se ha visto obligado a encargarse de un temario alejado de sus conocimientos e intereses, bien porque finalmente consigue dominar los contenidos. Por el contrario, cuando hablamos de un profesor que habitualmente (incluso a lo largo de su vida profesional) muestra poca EE se debe, normalmente, al hecho de que se cuestiona radicalmente su capacidad como maestro, es decir, como conjugando en sí mismo las dos dimensiones (lógico-informativa y físico-emocional), y por ello incapaz de transmitir la necesaria EE. 
comenzando siempre en la co-presencia física a través de capacidades neurológicas (que han emergido evolutivamente) que capacitan a las personas a presentar ellas mismas y recibir de las otras señales que permitan asumir mutuamente roles, emociones, culturas, estatus, atribuciones, etc., que generan dinámicas emocionales compartidas. De esos vínculos primeros se abrirá la posibilidad de generar relaciones que pueden mantenerse a distancia por procedimientos formalizadores impersonales. Pero la co-presencia física es imprescindible para la generación de vínculos emocionales fuertes (TURNER, 2013; TURNER; MARYANSKI, 2013). Ciertamente, el nivel de generación de emociones que permite la mediación técnica está condicionado culturalmente, dependiendo de las diferentes concepciones sobre lo que debe o no hacerse necesariamente a través de la copresencia o no. No obstante, esto no cuestiona el argumento general de que las emociones adquiridas a través de la co-presencia física son más fuertes, al menos en el actual nivel de desarrollo cerebral de la especie humana. Nuestra afirmación sigue siendo situacional $y$ contextual: no se niega que el desarrollo tecnológico pueda generar evolutivamente nuevas estructuras neuro-biológicas que hagan prescindible la co-presencia para la generación de vínculos emocionales fuertes. Pero en este nivel de evolución biológica, si de lo que se trata es de generar completos rituales educativos entre profesores y estudiantes, la co-presencia física es más adecuada que la virtualización completa.

Conclusión: la ambivalencia del nuevo entorno tecno-educativo: lo presencial y lo virtual

Como he puesto de manifiesto en la primera parte del artículo, los estudios actuales que tratan de realizar correlaciones entre las nuevas tecnologías y determinados aspectos educativos fallan muy a menudo en reconocer la ambivalencia y complejidad inherentes al nuevo entorno tecno-educativo. Las investigaciones que tratan de zanjar si la tecnología moderna es buena o mala para la educación o si aumenta o disminuye el éxito escolar, fallan en incorporar la ambivalencia como un rasgo constitutivo del ser humano y de todas sus creaciones históricas (ROMERO MOÑIVAS, 2015b). De ahí que nuestra reflexión no haya tratado sobre la incorporación o la eliminación de determinados artefactos tecnológicos (no hay educación sin algún tipo de mediación técnica), o de determinados saberes, o de determinados modelos de sistemas educativos, etc.; sino algo que debería de ser transversal a todo tipo de educación: la transmisión y adquisición mutua de energía emocional entre los profesores y los alumnos. Aunque esta energía emocional sólo puede ser adquirida de manera fuerte a través de la co-presencia física, eso no significa que se deban rechazar las propuestas de formación virtuales $y$ el uso de artefactos mediadores a distancia cuando los objetivos educativos son más limitados, como adquirir contenidos informativos o conseguir cualificaciones $y$ certificaciones, o como complemento de los rituales de co-presencia. De hecho, la adaptación a los nuevos tiempos es un rasgo de lo humano, siempre en continua evolución. Por esto, la propuesta de la co-presencia física y del profesor como catalizador de energía emocional no tiene como su modelo la mera presencialidad de la educación clásica, ni como su contra-modelo la actual educación virtualizada:

Lo primero, porque como ya he indicado, no basta la mera presencialidad del profesor para que se consiga la transmisión de la EE en el aula: la co-presencia física es una condición necesaria, pero no suficiente. El ejemplo más evidente es que desde los inicios de la educación hasta ahora la enseñaza generalmente ha sido presencial: sin embargo, muy pocos profesores universitarios han sido considerados maestros o, en general, pocos han alcanzado la excelencia docente en el sentido indicado anteriormente. Así, pues, el modelo del profesor como catalizador de energía emocional no tiene su fundamento en la clásica presencialidad -en la que es común que 
se produzca también la distancia transaccional de Moore (2007)-, sino que defiende algo más allá que un profesor en un aula transmitiendo información (mucha y obsoleta), como ha venido ocurriendo a menudo. La enseñanza en el aula tiene algo de teatral, y como toda representación teatral el objetivo es la emergencia de emociones compartidas entre actores y público. El profesor es como un actor que busca hacer partícipe de la obra a los estudiantes, para que no sean meros receptores pasivos del libreto.

Respecto a lo segundo, mi propuesta no tiene como enemigo a batir el nuevo entorno digital, su aprendizaje ubicuo, difuso, invisible, en movilidad, etc. (FERNÁNDEZ ENGUITA, 2013). Al contrario, este ecosistema o entramado socio-técnico-educativo es una nueva forma de comprender el mundo del conocimiento, de la enseñanza, del aprendizaje, de la transmisión de información, que permite una ventana más amplia con la que mirar el mundo, lejos, pues, de la tendencia a capturar el conocimiento en manos de unos pocos administradores de los saberes sagrados, o de lo que según ellos debe o no debe ser enseñado o aprendido, aquello en lo que hay que centrar la atención y lo que la distrae. Lo que he querido poner de manifiesto es que este nuevo entorno en su vertiente más virtualizada no parece capaz de transmitir aquellos otros contenidos intangibles que son más sólidamente mostrados a través de la copresencia física y de la convivencia personal, que dichos a través de lo lógico-argumentativo. En realidad, algo de esto ya es doctrina común incluso en algunos Master virtuales o cursos online, que suelen proponer algunas sesiones presenciales, reuniones o encuentros informales entre profesores y alumnos. Así, pues, el modelo de la co-presencia no invalida el nuevo entorno tecno-digital, sino que trata de situarlo en su ámbito apropiado, rescatando para sí lo que de bueno tiene en cuanto a descentralización, amplitud de información y ensanchamiento de la mirada al mundo.

Por ello, siempre hay que preguntarse qué objetivos busca una persona cuando se matricula en unos estudios formalizados. Es significativo que muchos alumnos de la UNED (Universidad Nacional de Educación a Distancia en España), con más edad de la habitual para iniciar estudios y con más obligaciones personales, familiares y laborales, saquen tiempo para poder ir a las clases presenciales que les llenan, que les motivan luego para poder estudiar solos y para adquirir EE a través de las interacciones con profesores y compañeros. Por supuesto, si el objetivo es tener un título o acumular determinadas informaciones, no es necesario este tipo de rituales físicos. Y luchar contra eso sería tan irracional como luchar contra la lectura sosegada y solitaria de libros tradicionales. Pero hay otros objetivos que buscan los estudiantes que tienen que ver precisamente con su constitución como personas y su adquisición de pasiones vitales que sólo pueden lograrse a través de la corporalidad de las asambleas rituales físicas. A fin de cuentas, estamos muy lejos del pernicioso dualismo platónico, que parecía condenar la carne a pura cárcel de lo anímico. No hay angelismos descarnados. Las personas necesitamos la corporalidad para transmitir lo más básico de nuestra naturaleza: los sentimientos, las emociones, lo interpersonal, los deseos y las esperanzas. Por ello, no considero que pueda temerse que la docencia presencial desparezca de la universidad. Sospecho, eso sí, que muchas de las tecnofobias de algunos profesores radican, precisamente, en que la auto-percepción de $s u$ eficacia es muy baja. Temen ser reemplazados por un ordenador, quizá porque involuntariamente reconocen que, de hecho, su forma de ser profesor podría ser radicalmente mejorada por una máquina. De ahí el sorprendente miedo de algunos profesores a que sus alumnos tengan ordenadores en las aulas y puedan controlar lo que dicen con un sólo vistazo en Google. Evidentemente, un profesor que sólo transmite información (y mucha de ella obsoleta o tendenciosa por falta de investigación) no genera rituales de interacción exitosos, y el alumno lo percibe. Es un ritual fracasado, como una obra de teatro que no genera aplausos del público porque no se ha sentido transportado emocionalmente. 
Quizá sea esta la causa del por qué algunos docentes, especialmente en la universidad, son tan reacios a la virtualización de algunos ámbitos educativos. Un profesor que genera EE en el aula y que transmite además de contenidos esos otros rasgos que hacen de él un buen profesor en el sentido arriba indicado, no suele ser tecnofóbico. $\mathrm{Al}$ contrario, es habitual que él mismo utilice esas herramientas en lo que se refiere a la transmisión de información e incluso para la autonomía investigadora del estudiante. Un profesor así sabe que no es probable que la educación universitaria en bloque sea completamente virtualizada o que las tecnologías permitan que los alumnos, sin profesores, puedan adquirir todo el conocimiento. Es consciente de que el carácter profundamente corporal y ritual de la educación impide ese proceso desde un punto de vista macrosocial. Por supuesto, que haya universidades o centros de formación completamente virtuales no supone que toda la educación lo pueda ser algún día. Por ello, me parece que en este caso concreto, el profesor universitario debe estar más preocupado de adquirir EE y de saber ser catalizador de esa EE para sus alumnos, que de alinearse dogmáticamente entre las filas de tecnófobos y tecnófilos, actitud que lejos de ser científica es profundamente irracional.

\section{Referencias}

BANDURA, Albert. Self-efficay: Toward a unifying theory of behavioral change. Psychological Review, Washington, n. 84, p. 191-215, 1977.

BANDURA, Albert; PAJARES, Frank. Prólogo. In: PRIETO NAVARRO, Leonor. Autoeficacia del profesor universitario: eficacia percibida y práctica docente. Madrid: Narcea, 2007.

COLLINS, Randall. The sociology of philosophies: a global theory of intellectual change. Cambridge: Harvard University Press, 1998.

COLLINS, Randall. Interaction Ritual Chains. Princeton: Princeton University Press, 2004.

DAMASIO, Antonio. El error de Descartes. Barcelona: Crítica, 2006.

DEGELE, Nina. Einführung in die techniksoziologie. München: Fink, 2002.

ELIAS, Norbert. Was ist Soziologie? München: Juventa Verlag, 1970.

FERNÁNDEZ ENGUITA, Mariano. El aprendizaje difuso y el declive de la institución escolar. Revista de la Asociación Española de Sociología de la Educación, Madrid, v. 6, n. 2, p. 150-167, 2013.

GOFFMAN, Irving. La presentación de la persona en la vida cotidiana. Buenos Aires: Amorrortu, 1994.

HOLMBERG, Börje. Distance education in essence. Oldenburg: Bibliotheks- und Informationssysem der Universität Oldenburg, 2003.

HUGHES, Thomas. El impulso tecnológico. In: ROE SMITH, Merrit; MARX, Leo (Ed.). Historia y determinismo tecnológico. Madrid: Alianza, 1996. p. 117-130.

HUGHES, Thomas. Human-built world: how to think about technology and culture. Chicago: London: The University of Chicago Press, 2004.

HUGHES, Thomas. The evolution of large technological systems. In: PINCH, Trevor J.; HUGHES, Thomas P.; BIJKER, Wiebe E. The social construction of technological systems: new directions in the sociology and history of technology. Massachusetts: MIT Press, 1997. p. 51-82.

JOERGES, Berndward. Technik, das andere der gesellschaft?. In: ROPOHL, Günter (Hg.). Erträge interdisziplinärer technikforschung: Eine bilanz nach 20 Jahren. Berlin: E. Schmidt Verlag, 2001. p. 165-180.

LATOUR, Bruno. Ciencia en acción: cómo seguir a los científicos y a los ingenieros a través de la sociedad. Barcelona: Labor, 1992. 
LATOUR, Bruno. Nous n’avons jamais été modernes: essai d’anthropologie symétrique. Paris: La Decouverte, 1997.

LAW, John. Notes on the theory of the actor-network: ordering, strategy, and heterogeneity. Systemic Practice and Action Research, v. 5, n. 4, p. 379-393, 1992.

MÈLICH, Joan-Carles. Ética de la compasión. Barcelona: Herder, 2010.

MOORE, Michael Graham. The theory of transactional distance. In: MOORE, Michael Grahame; ANDERSON, William G. (Ed.). Handbook of distance education. London: Mahwah: Lawrence Erlbaum Associates, 2007. p. 89-108.

MOORE, Michael Graham; KEARSLEY, Greg. Distance education: a systems view. Belmont: Wadsworth, 2005.

PAJARES, Frank. Self-efficacy beliefs in academic settings. Review of Educational Research, Washington, v. 62, p. 307-332, 1996.

PETERS, Otto. Distance education in transition: new trends and challenges. Oldenburg: Bibliotheks- und Informationssystem der Universität Oldenburg, 2004.

PETERS, Otto. Learning and teaching in distance education. London: Kogan Page, 1998.

PINCH, Trevor; HUGHES, Thomas; BIJKER, Wiebe. The social construction of technological systems: new directions in the sociology and history of technology. Massachusetts: MIT Press, 1997.

PRIETO NAVARRO, Leonor. Autoeficacia del profesor universitario: eficacia percibida y práctica docente. Madrid: Narcea, 2007.

RAMMERT, Werner. Die teshnische konstruktion als teil der gesellschaftlichen konstrucktion der wirklichkeit. Technical University Tecnhology Studies Working Papers, Berlin, 2002.

ROMERO MOÑIVAS, Jesús. Del aprendizaje difuso al aprendizaje situacional: una explicación estructural-conflictiva de las relaciones entre la tecnología y la educación en la sociedad de la información. Revista de la Asociación Española de Sociología de la Educación, Madrid, v. 6, n. 2, p. 210-227, 2013b.

ROMERO MOÑIVAS, Jesús. Hacia una comprensión micro-situacional de la ambivalencia entre libertad humana y entornos artificiales. Argumentos de Razón Técnica, Sevilla, n. 18, 2015. En imprenta.

ROMERO MOÑIVAS, Jesús. La ambivalencia humana y sus implicaciones para la sociología. En imprenta.

ROMERO MOÑIVAS, Jesús. Los fundamentos de la sociología de Norbert Elias. Valencia: Tirant lo Blanch, 2013a.

ROMERO MOÑIIVAS, Jesús. Micro-rituales situacionales y macro-dinámicas geopolíticas: una aproximación global a la sociología de Randall Collins. Revista Española de Sociología, Madrid, n. 20, p. 63-103, 2013b.

STAUDENMAIER, John. Technology's storytellers: reweaving the human fabric. Massachusetts: MIT Press, 1985.

TASSEY, Gregory. The technology imperative. Massachussets: Edward Elgar, 2007.

TURNER, Jonathan $\mathrm{H}$. Neurology and interpersonal behavior: the basic challenge for neurosociology. In: FRANKS, David; TURNER, Jonathan (Ed.). Handbook of neurosociology. New York: Springer, 2013. p. 119-137.

TURNER, Jonathan H.; MARYANSKI, Alexandra. The evolution of the neurological basis of human. sociality. In: FRANKS, David; TURNER, Jonathan (Ed.). Handbook of neurosociology. New York: Springer, 2013. p. 289-309.

Recibido en: 13.01.2015

Aprobado en: 14.04.2015

Jesús Romero Moñivas es doctor en sociologia, profesor en la Universidad Complutense de Madrid y en la Universidad Nacional de Educación a Distancia (Madrid, España). Autor de 40 publicaciones de diversas temáticas con orientación interdisciplinar. (Para acceder a sus publicaciones: www.jesromero.wordpress.com). 